\title{
Hybrid inorganic/organic photonic crystal biochips for cancer biomarkers detection
}

\author{
Alberto Sinibaldi ${ }^{1}$, Norbert Danz ${ }^{2}$, Peter Munzert ${ }^{2}$, and Francesco Michelotti ${ }^{1}{ }^{*}$ \\ 1. SAPIENZA Università di Roma, Department of Basic and Applied Sciences for Engineering, \\ Via A. Scarpa, 16, 00161 Roma, Italy \\ 2. Fraunhofer Institute for Applied Optics and Precision Engineering, A.-Einstein-Str. 7, 07745 \\ Jena, Germany
}

\begin{abstract}
We report on hybrid inorganic/organic one-dimensional photonic crystal biochips sustaining Bloch surface waves. The biochips were used, together with an optical platform operating in a label-free and fluorescence configuration simultaneously, to detect the cancer biomarker Angiopoietin 2 in a protein base buffer. The hybrid photonic crystals include a thin functionalization poly-acrylic acid layer deposited by plasma polymerization, which is used to immobilize a monoclonal antibody for highly specific biological recognition. The fluorescence operation mode is described in detail, putting into evidence the role of field enhancement and localization at the photonic crystal surface in the shaping and intensification of the angular fluorescence pattern. In the fluorescence operation mode, the hybrid biochips can attain the limit of detection $6 \mathrm{ng} / \mathrm{ml}$.
\end{abstract}

Keywords: Sensors, one-dimensional Photonic Crystals, Bloch Surface Waves.

Corresponding author

Email: francesco.michelotti@uniroma1.it 


\section{Introduction}

Bloch surface waves (BSW) propagating at the interface between a one dimensional photonic crystal (1DPC) and an external medium [1,2] have been suggested for label-free biosensors [3,4]. When used in a classical Kretschmann-Raether [5] total internal reflection (TIR) configuration, similar to surface plasmon resonance (SPR) sensing schemes [6], the BSW biochips feature very low angular resonance widths compared to that of SPR, due to the low losses in the dielectric stacks [7].

The optimization of the 1DPC stack, in terms of geometry and materials, offers the possibility to improve the label-free limit of detection (LoD) [8]. However, the strong field localization and enhancement at the 1DPC surface favor utilizing BSW coupled fluorescence emission [9]. Coupling the label-free operation to a second detection scheme relying on the emission of fluorescent labels can lead to a complementary analysis and a decrease of the LoD [10,11].

We report here on the application of a combined label-free and fluorescence biosensing platform [12] to the detection of the Angiopoietin-2 cancer biomarker in buffer solutions. The platform makes use of disposable biochips fabricated by depositing 1DPC onto injection molded plastic substrates. Here the 1DPC include an inorganic multilayer and an organic nanometric top layer providing the chemical functionalization of the biosensor's surface.

\section{BSW biochips}

The geometry of the 1DPC used in this study is shown in Figure 1(a). A dielectric multilayer was directly deposited on a prismatic organic substrate (TOPAS, $\mathrm{n}_{\mathrm{s}}=1.530$ ) by plasma ion assisted deposition (PIAD) under high vacuum conditions [13]. Then the multilayer was coated with an organic poly-acrylic acid (ppAA) functional thin film by a plasma polymerization technique and making use of a plasma enhanced chemical vapour deposition reactor [14]. The geometry of the underlying inorganic part of the 1DPC was designed in a way that, after adding the organic functional film/layer, the characteristics of the overall 1DPC are optimized for both label-free and fluorescence sensing.

Besides being used to tune the BSW biochip performance [15], the thin organic functional layer permits to covalently graft and immobilize the proteins that are needed to provide the specificity of the biosensor. ppAA thin coatings are a convenient choice for surface chemical functionalization since they do not affect the chemical properties of the biosensors, are conformal, do not need any 
specific surface pre-treatment for adhesion and can provide different density of functional groups, wettability and chemical stability by simply varying the deposition process parameters [16].

The inorganic part of the $1 \mathrm{DPC}$ is constituted of $\mathrm{SiO}_{2}, \mathrm{Ta}_{2} \mathrm{O}_{5}$ and $\mathrm{TiO}_{2}$, with complex refractive indices: $\mathrm{n}\left(\mathrm{SiO}_{2}\right)=1.474+\mathrm{j} 5 \times 10^{-6}, \mathrm{n}\left(\mathrm{Ta}_{2} \mathrm{O}_{5}\right)=2.160+\mathrm{j} 5 \times 10^{-5}, \mathrm{n}\left(\mathrm{TiO}_{2}\right)=2.28+\mathrm{j} 1.8 \times 10^{-3}$ at $\lambda_{0}=670 \mathrm{~nm}$ [13]. Starting from the plastic substrate, the multilayer is constituted by a first $\mathrm{SiO}_{2}$ layer, which improves the reliability of the overlaying 1DPC, two $\mathrm{Ta}_{2} \mathrm{O}_{5} / \mathrm{SiO}_{2}$ bilayers and a top $\mathrm{TiO}_{2}$ layer. The thicknesses of the layers are: $\mathrm{d}\left(\mathrm{SiO}_{2}\right)=275 \mathrm{~nm}, \mathrm{~d}\left(\mathrm{Ta}_{2} \mathrm{O}_{5}\right)=120 \mathrm{~nm}$, and $\mathrm{d}\left(\mathrm{TiO}_{2}\right)=$ $20 \mathrm{~nm}$.

The thickness of the ppAA thin topping layer was estimated by atomic force microscopy and is $\mathrm{d}(\mathrm{ppAA}) \sim 40 \mathrm{~nm}$ and the refractive index is $\mathrm{n}(\mathrm{ppAA})=1.52$, obtained by spectroscopic ellipsometry [14].

a)

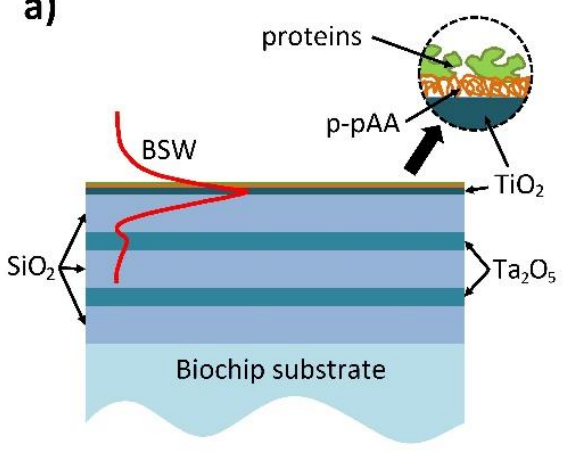

b)

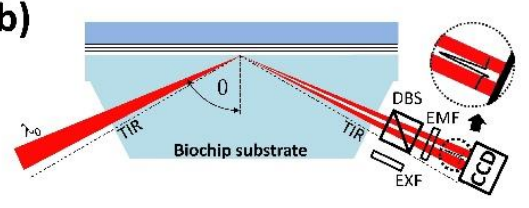

c)

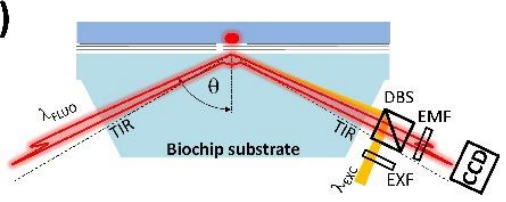

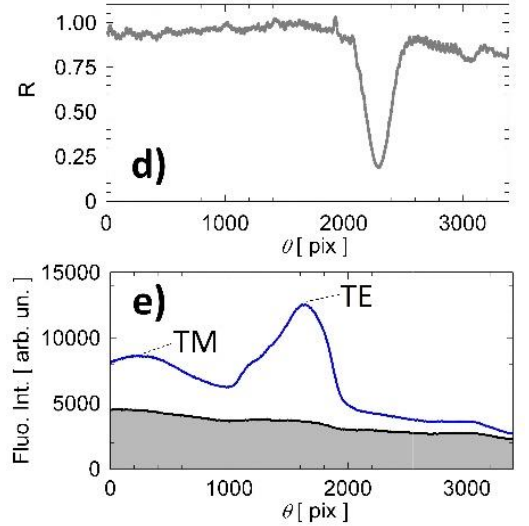

Figure 1: (a) 1DPC geometry including the polymer functional ppAA film (not to scale). Sketches of the label-free (b) and fluorescence (c) modes of operation. (d) TIR angular reflectance profile, with the biochip in buffer. (e) Fluorescence emission at the end of an assay where Ang2 was detected (blue curve) and before starting the experiment (black curve).

After chemical functionalization and bio-conjugation, a plastic cover with a soft polymer layer defining a fluidic channel ( $800 \mu \mathrm{m}$ wide, $100 \mu \mathrm{m}$ high, and $27 \mathrm{~mm}$ long) is clicked on top of the biochip and permits the injection of fluids for the analysis [12].

\section{Read-out configuration}

The BSW biochips are mounted in an optical platform and are used in either a label-free or in a fluorescence mode of operation, as shown schematically in Figure 1(b) and Figure 1(c), 
respectively. For the sake of simplicity, in the figures, we do not show the optical components that are used to focus and image the light beams; for a complete description of the optical layout of the platform one can refer to our precedent work [12]. In particular, the optical system is based on the use of cylindrical optics and can simultaneously perform measurements in several different spots aligned along a line over the biochip surface. The rows and columns of the CCD camera (Apogee Ascent with Sony ICX814 sensor) are then used, respectively, for either angular imaging (3388 pixel) or spot imaging (2712 pixel).

In the label-free operation, the BSW biochip is illuminated through a prism coupler in TIR conditions $[6,17]$ by a TE polarized and focused (FWHM above $4^{\circ}$ ) laser beam at the wavelength $\lambda_{0}=670 \mathrm{~nm}$. The reflected beam is imaged on the CCD. As an example, in Figure 1(d) we show the TIR spectrum as a function of the incidence angle inside the prism coupler $\theta$, in a $2.9^{\circ}$ angular range (sampled by 3388 pixel). Excitation of a BSW gives rise to a dip in the angular reflectance spectrum at $\theta_{0, \mathrm{BSW}}$. The $1 \mathrm{DPC}$ was designed to operate at $\theta_{0, \mathrm{BSW}}=69^{\circ}$ and the thin $\mathrm{TiO}_{2}$ layer features were tuned to get a narrow width and large depth of the resonant dip.

Figure 1(a) illustrates (red curve) the distribution of the TE polarised BSW energy density calculated at $\lambda_{0}$ and in resonance conditions. The BSW is confined at the interface between the 1DPC and the external medium, where the field decays exponentially with a penetration length $\mathrm{L}_{\mathrm{pen}}$ $=117 \mathrm{~nm}$. Given its strong localization, the BSW is very sensitive to refractive index perturbations in the vicinity of the $\mathrm{TiO} 2$ layer and can be used to monitor protein binding events at the biochip surface, as revealed by the shift of the resonant dip.

In the fluorescence operation, the BSW biochip is excited in TIR conditions at an angle $\theta_{\text {EXC }}$ by a TE polarized and slightly focused $\left(\mathrm{FWHM}=0.64^{\circ}\right)$ laser beam at the wavelength $\lambda_{\mathrm{EXC}}=635 \mathrm{~nm}$, matching the absorption peak of the specific dye label that we selected for the biological assays (Dylight 650). The effect of the BSW excitation on fluorescence emission is two-fold. On one-hand, if $\theta_{\text {EXC }}$ is such that a resonant excitation condition of a BSW at $\lambda_{\text {EXC }}$ is achieved, the excitation intensity is enhanced at the 1DPC surface, giving rise to an increased fluorescence emission. On the other hand, the emission at $\lambda_{\mathrm{EM}}>\lambda_{\mathrm{EXC}}$ of the dye-labelled biomolecules possibly captured and excited at the biochip surface, given the large BSW local density of the states [18], is channelled to the BSW modes, which then are radiated through the substrate, as shown in Figure 1(c).

As an example, Figure 1(e) shows the angular emission spectrum recorded by the CCD in a $8^{\circ}$ angular range; such a wider range is obtained by inserting a cylindrical zoom lens in the detection system. Given that the 1DPC sustains both TE and TM polarized BSW, with different dispersions, 
the emission is characterized by two angularly separated TE and TM bands. For each band, each angle corresponds to a different wavelength inside the emission spectrum of Dylight 650. In Figure 1(e), the black curve is the measurement for an unlabelled biochip, and accounts for the background signal captured by the CCD due to either stray light from the excitation beam or intrinsic BSW coupled fluorescence of the 1DPC materials. In all experiments reported below such a background was always subtracted from the fluorescence signal in the presence of labels, which is given by the blue curve shown in Figure 1(e) as an example.

\section{Bio-conjugation procedure and assay format}

The ppAA coated 1DPC (Fig. 2a) were chemically treated (15 min) to activate the -COOH groups with a solution of sulfo-NHS (N-hydroxysulfosuccinimide, Thermo Scientific) and EDC (1-ethyl-3(3-dimethylaminopropyl)carbodiimide hydrochloride, Thermo Scientific) in a BupH MES (2-(Nmorpholino)ethanesulfonic acid, Thermo Scientific) buffer at $\mathrm{pH} 4.7$ (Fig. 2b), followed by a washing step in BupH MES and Dulbecco's Phosphate Buffer Saline buffer (referred to as "B" below) at $10 \mathrm{mM}(\mathrm{D}-\mathrm{PBS} 1 \mathrm{X})$.

Two separated signal and reference regions were defined on the activated-ppAA 1DPC by means of a hydrophobic marker, where either protein $\mathrm{G}(\mathrm{PtG}, 0.5 \mathrm{mg} / \mathrm{ml}$, Thermo Scientific) or bovine serum albumin (BSA, $10 \mathrm{mg} / \mathrm{ml}$, Sigma-Aldrich), dissolved in D-PBS 1X, were incubated for $1 \mathrm{~h}$ at ambient temperature (AT), respectively (Fig. 2c). Subsequently, the biochip was immersed in a BSA solution (10 mg/ml in B) to block the remaining reactive sites (overnight at $4{ }^{\circ} \mathrm{C}$ ). At the end, on the biochip surface there are a signal region (PtG), which is capable to bind and orient the capture antibodies, and a reference region (BSA), which is biochemically inert (Fig. 2d). Immediately before their use in a detection assay, the surface of the biochips was treated with a regeneration solution made of glycine (20 mM, Sigma-Aldrich) in deionized water and hydrogen chloride $(\mathrm{HCl})$ with a $\mathrm{pH}$ of 2.5 for $10 \mathrm{~min}$ at $\mathrm{AT}$, to remove any adlayer formed during the BSA blocking step.

In the sandwich assays the target molecule was a Recombinant Human Angiopoietin 2 (Ang2, 623AN, R\&D Systems) spiked in a modified buffer, acting as a protein base buffer, consisting in a solution of D-PBS $1 \mathrm{X}+0.1 \mathrm{wt} \% \mathrm{BSA}$ (referred to as "MB" below). To specifically detect the presence of Ang2, a monoclonal anti-human Ang2 antibody (a-Ang2, MAB0983, R\&D Systems) was used as a capturing element (Fig. 2e). After the interaction with the Ang2 sample (Fig. 2f), a specific biotinylated-detection Anti-Ang2 antibody (a-Ang2*, BAF623, R\&D Systems) binds to 
Ang2 and provides biotinylated characteristics to the signal region (Fig. 2g). Finally, labelling takes place by the interaction with a solution of neutravidin labelled with fluorophores emitting at $\lambda_{\mathrm{EM}}=$ 670 nm (NeutrAvidin DyLight 650, N650, ThermoScientific). Neutravidin was selected for its high affinity to biotin, therefore enabling detection of the a-Ang2*/Ang2 complex (Fig. 2h).
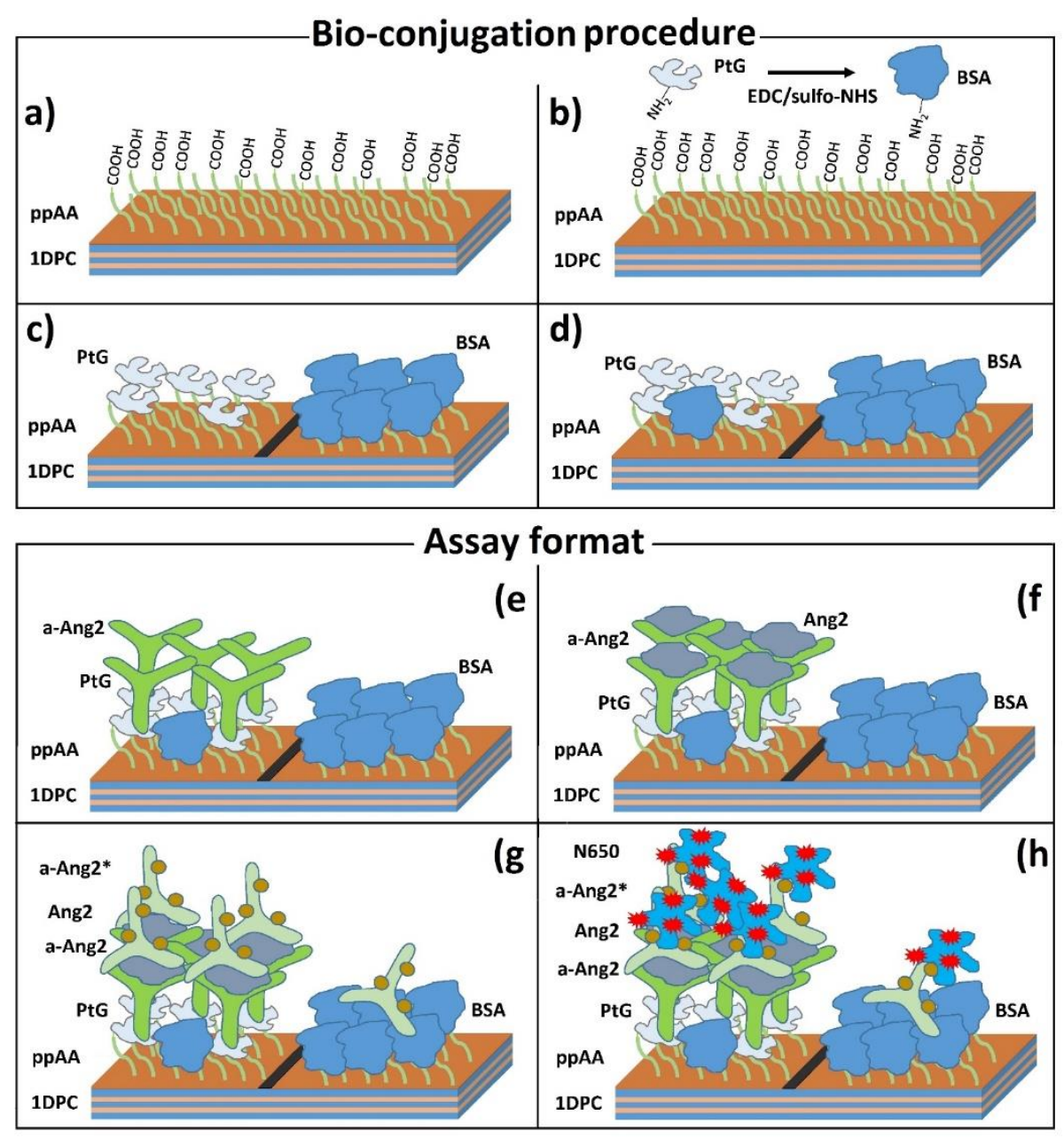

Figure 2: $(\mathrm{a} \rightarrow \mathrm{d})$ Subsequent steps of the direct approach for the bio-conjugation of proteins (PtG, BSA) to the ppAA-loaded 1DPC surface prior to the assay. $(e \rightarrow h)$ Sandwich assay format used in all experiments performed in this work.

\section{Results}

In Figure 3 we show the sensograms recorded in label-free mode during an Ang2 cancer biomarker detection assay. The angular position of the BSW resonant dip $\theta_{0, \mathrm{BSW}}$ is tracked as a function of time. The two curves are the result of averaging the signals measured in 7 different spots in either the signal region $(\mathrm{PtG})$ or the reference region (BSA). Each spot corresponds to $100 \mathrm{CCD}$ camera rows. 
The assay starts in MB and proceeds according to the following steps. First we inject a solution of a-Ang2 (30 $\mu \mathrm{g} / \mathrm{ml}$ in $\mathrm{MB})$ and incubate for $25 \mathrm{~min}$. After such a step, the biochip is ready to be used to detect the biomarkers. In the specific case of Figure 3, we injected a solution of Ang2 (50 ng/ml in MB) and incubated for $50 \mathrm{~min}$. This ends up the label-free part of the assay. For the subsequent fluorescence part of the assay, we inject a solution of a-Ang2* $(2 \mu \mathrm{g} / \mathrm{ml}$ in MB) and incubate for 27 min. Then, we change the running buffer from MB to B and inject a solution of $\mathrm{N} 650(10 \mu \mathrm{g} / \mathrm{ml}$ in B) and incubate for $13 \mathrm{~min}$. For all analytes, we injected $100 \mu \mathrm{l}$ of the solutions and recirculated 50 $\mu \mathrm{l}$ by pumping back and forth. All incubation steps are followed by a washing step carried out by injecting $500 \mu \mathrm{l}$ of either MB or B. In all cases the injection flow rate was $4 \mu \mathrm{l} / \mathrm{s}$.

Figure 3(a) shows a drift of the label-free signals in both the reference and signal regions, due to changes of the temperature, pressure and laser wavelength $\lambda_{0}$. Such drifts are ruled out by subtracting the two curves, giving rise to the differential signal shown in Figure 3(b). Besides giving access to label-free detection of the Ang2 biomarker (if concluded), the differential signal permits to monitor all phases of the subsequent fluorescence assay, which is not possible in common techniques, such as ELISA for example. In particular one can monitor the correct immobilization of the capture antibodies, the quality and stability of the blocking agents, the presence of biochip defects or of fluid flow anomalies.

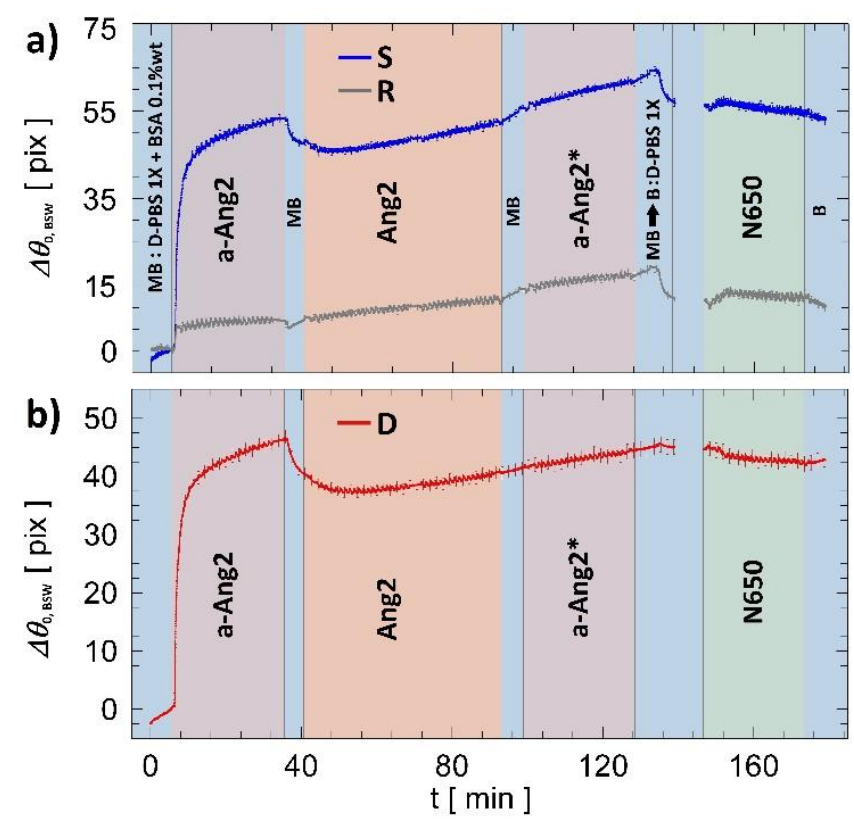

Figure 3: (a) Label-free sensograms recorded during an Ang2 sandwich assay in the signal (S, blue) and reference (R, grey) regions (average of 7 spots). The error bars are the standard error of the mean. (b) The red curve is the differential signal with error bars every $2 \mathrm{~min}$. 
For what is concerning the fluorescence assay, in Figure 4(a) we show the angular emission spectra recorded at resonant excitation for the Ang2 detection assay described above (50 $\mathrm{ng} / \mathrm{ml} \mathrm{in} \mathrm{MB})$. Fluorescence emission in B was measured in the two signal (blue data) and reference regions (grey data), after incubating the N650 solution and washing. The curves were obtained by averaging the signals in the same 7 spots used for the label-free measurement. In both cases the spectra were corrected by subtracting the background fluorescence (similar to Figure 1(e)) measured immediately before injecting the N650 solution and averaged on the same spots.

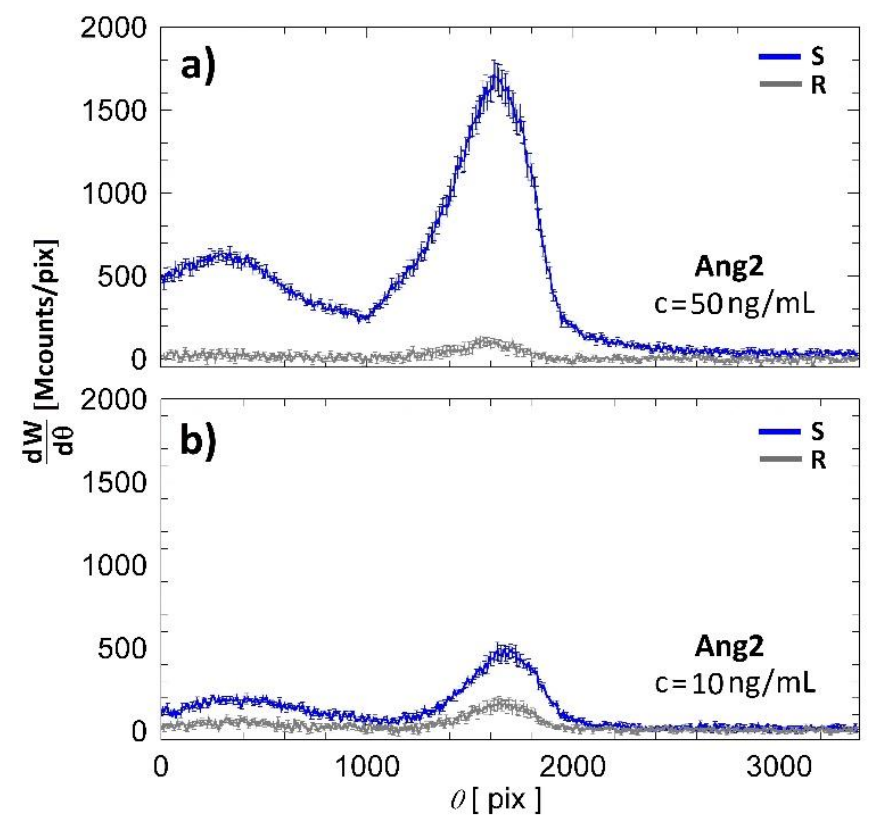

Figure 4: Average fluorescence emission signals from the labelled antibody-antigen complexes at the 1DPC surface for the signal (S, blue) and reference ( $\mathrm{R}$, grey) regions (7 spots). The fluorescence signals were recorded at the end of a $50 \mathrm{ng} / \mathrm{ml}$ (a) and a $10 \mathrm{ng} / \mathrm{ml}$ (b) Ang2 sandwich assay. The error bars are the standard error of the mean.

In all fluorescence assays, the angular emission spectrum under resonant excitation conditions is determined according to the following procedure. The incidence angle of the fluorescence excitation laser $\theta_{\text {EXC }}$ is scanned in a convenient angular range around the BSW resonant dip $\theta_{\text {EXC,BSw }}$ at $\lambda_{\text {EXC }}$ and fluorescence images are acquired in a sequence by the CCD camera during the scan. Thanks to the dispersion of the BSW mode, $\theta_{\mathrm{EXC}, \mathrm{BSW}}>\theta_{0, \mathrm{BSW}}$ and lays outside the CCD angular field of view (right hand side in Figure 4). In the platform, scanning of $\theta_{\mathrm{EXC}}$ is achieved by laterally shifting the fluorescence excitation laser while keeping fixed the focusing lens (not shown in Figure 1). 


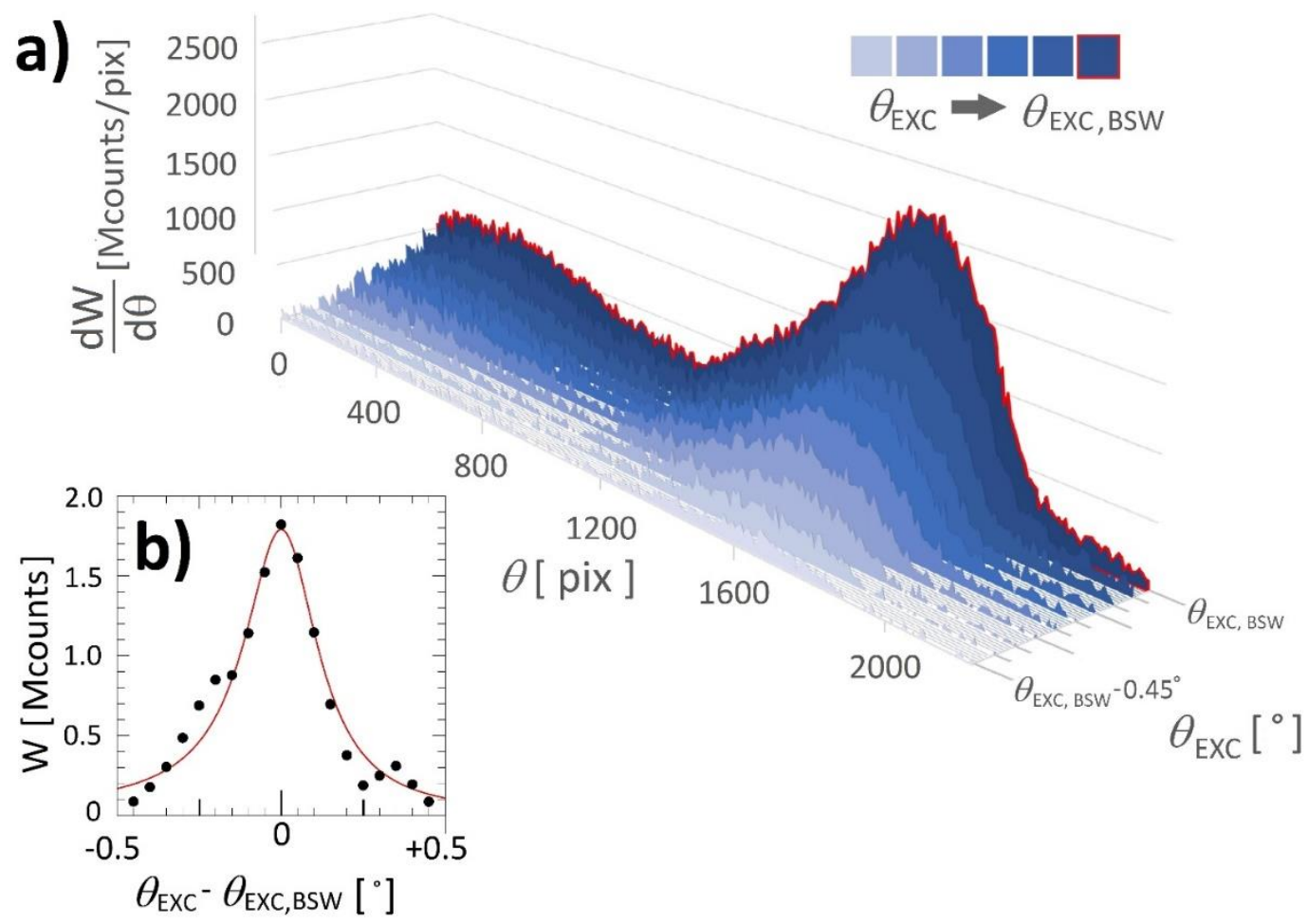

Figure 5: (a) Background subtracted fluorescence intensity at the end of a $50 \mathrm{ng} / \mathrm{ml} \mathrm{Ang2} \mathrm{assay} \mathrm{for}$ several different $\theta_{\text {EXC }}$ of the fluorescence excitation laser at $\lambda_{\text {EXC }}$. The maximum fluorescence

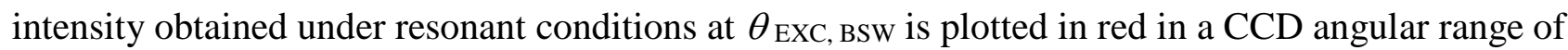
$[0,2200]$ pixels. (b) Integrated fluorescence intensity in proximity of the $\theta_{\mathrm{EXC}}$, BSW. and Lorentzian fit (red curve).

As an example, in Figure 5a we show a set of angular emission spectra recorded for different values of $\theta_{\mathrm{EXC}}$, from off-resonance to resonance. The background subtracted spectra refer to the Ang2 detection assay at $50 \mathrm{ng} / \mathrm{ml}$ and to one single spot within the signal region on the biochip. Clearly, when approaching $\theta_{\text {EXC }}(\mathrm{BSW})$ the intensity of the angular emission increases up to a maximum (red curve), due to the BSW intensity enhancement at the 1DPC surface. This is confirmed by Figure 5b, which shows that the total BSW emitted power, i.e. the integral of the curves in Figure $5 \mathrm{a}$, is peaked around $\theta_{\mathrm{BSW}, \mathrm{EXC}}$. For that specific spot, we therefore assign as a fluorescence angular spectrum under resonant excitation the maximum curve (red) among those collected for that spot during the scan.

Owing to the inhomogeneities of the bare 1DPC and to the different degree of binding, resonant excitation can take place in each spot at a different $\theta_{\mathrm{BSW}, \mathrm{EXC}}$. Therefore, the same procedure is repeated by analyzing the series of CCD images and selecting for each spot the most intense angular 
emission spectrum. Finally, we obtain a synthetic CCD image, which is used, by averaging over a given number of spots in the signal and reference regions, to generate the curves shown in Figure 4a. During such a procedure, the value of $\theta_{0, \mathrm{BSw}}$ for each spot that is extracted from the label-free measurement is used to determine, spot by spot and making use of the BSW dispersion, the position of the $\theta_{\text {EXC }}$ scanning window.

In Figure 4b, we show the results for an Ang2 detection assay at $10 \mathrm{ng} / \mathrm{ml}$ in $\mathrm{MB}$, obtained according to the same procedures. Also in this case the response of the signal and reference regions can be clearly distinguished in the limit of the measurement noise.

\section{Discussion}

The sensograms shown in Figure 3 indicate that label-free detection of Ang2 at $50 \mathrm{ng} / \mathrm{ml}$ cannot be concluded. The growth of the differential signal during the Ang2 solution incubation cannot be distinguished from the residual slope, which characterizes the whole sensogram. Such a residual signal could be due to BSA from the MB binding non-specifically to the biochip surface. The same is true, obviously, also for the assay at $10 \mathrm{ng} / \mathrm{ml}$ (not shown). Therefore, the label-free LoD of the present ppAA based BSW biochips is surely larger than $50 \mathrm{ng} / \mathrm{ml}$. Such a limitation is also related to the efficiency of the chemical surface functionalization. As a comparison, the same BSW biochips with a different surface chemistry, based on 3-Aminopropyl)triethoxysilane (APTES) with glutaraldehyde $(\mathrm{GAH})$ as cross-linker [19] showed the same limitation for label-free Ang2 detection [20], whereas, in case the PtG immobilization step is dropped, they were demonstrated to label-free detect a different biomarker (VEGF) at $50 \mathrm{ng} / \mathrm{ml}$ with an estimated LoD of $11.5 \mathrm{ng} / \mathrm{ml}$ [12].

Despite the limitations, the label-free signal provides useful real-time information on the assay development. Figure 3 shows that PtG is efficiently binding the capture antibody in the signal region of the biochip, whereas BSA blocking guarantees a good anti-fouling action in the reference region. From the differential shift of the BSW resonance after the a-Ang2 incubation one can evaluate, making use of the label-free sensitivity of the biochips [11], that the a-Ang2 surface density is $(65 \pm 2) \mathrm{ng} / \mathrm{cm}^{2}$. The residual slope that is observed in the MB, in which the BSA concentration is very large (about $1 \mathrm{mg} / \mathrm{ml}$ ), indicates that blocking could be improved by using different and smaller passivation agents, such as caseine for example. The sensorgrams also show that there are no instabilities of the microfluidic environment (bubbles, leaks, blockage of the microchannel), guaranteeing homogeneity during the assay steps (injection, recirculation, washing). It is therefore clear that Ang2 detection at low concentration with the present platform needs to be accessed by fluorescence detection. 
Figure 4 shows that Ang2 detection in the fluorescence mode is achieved in both assays, at $50 \mathrm{ng} / \mathrm{ml}$ and $10 \mathrm{ng} / \mathrm{ml}$. The intensity in the signal region is well distinguished from that in the reference region. Such a result is obtained thanks to the BSW enhanced fluorescence operation. Figure 5(b) shows that the intensity of the fluorescence emission is increased by a factor 45 when operating at resonant excitation. Figure 4 shows that the fluorescence collection efficiency is improved due to the fact that the emission is confined in an angular interval of about $5^{\circ}$.

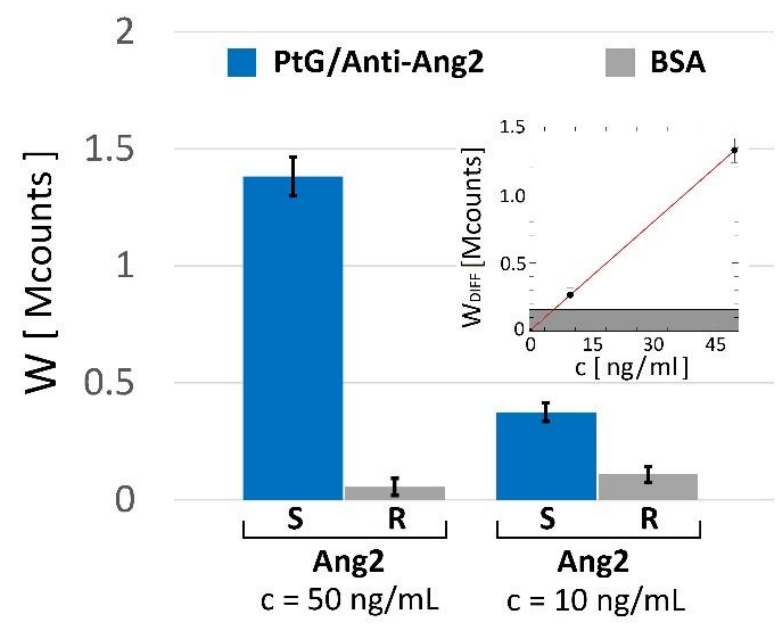

Figure 6: (a) Bar plot of the integrated fluorescence intensities $\mathrm{W}$ for both assays. In the inset it is represented the linear dependence of $\mathrm{W}_{\mathrm{DIFF}}$ as a function of the Ang2 concentration.

The result of the assays is better evidenced in Figure 6, where the bar plot shows the experimental values of $\mathrm{W}$ for both assays. We conclude that Ang2 was detected at $10 \mathrm{ng} / \mathrm{ml}$, within the error

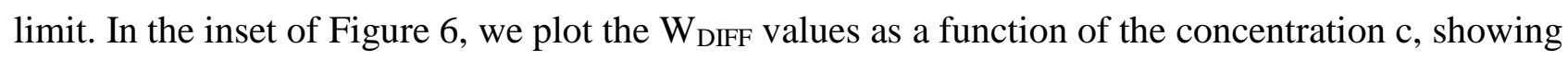
that $\mathrm{W}_{\mathrm{DIFF}}$ scales linearly with $\mathrm{c}$, which is expected in the limit of low concentration. Such a plot can be used to retrieve an estimation of the fluorescence LoD, by extrapolating the linear dependency down to the level corresponding to three times the standard deviation of the measurement at lowest c. We find that the fluorescence LoD is $6 \mathrm{ng} / \mathrm{ml}$. Again such a value can be compared to that obtained with the same biochips and APTES chemistry and without PtG, which is $0.65 \mathrm{ng} / \mathrm{ml}$ for the same Ang2 biomarker [21].

The results obtained in both the label-free and fluorescence mode of operation seem to confirm that, using the same BSW biochips, the immobilization protocol based on ppAA/PtG/a-Ang2 is less efficient that a protocol based on APTES/GAH/a-Ang2.

\section{Conclusions}


In conclusion we demonstrated that the hybrid ppAA/inorganic BSW biochips can be efficiently used to detect the Ang2 biomarker at low concentration in a modified buffer, when operating the platform in the fluorescence mode where BSW coupled fluorescence excitation and emission is exploited. In particular, the plasma polymerized poly-acrylic acid organic top layer is shown to immobilize PtG and the capture antibody at the surface of the biochips, with a surface density of the antibodies as large as $(65 \pm 2) \mathrm{ng} / \mathrm{cm}^{2}$. In the test assays, the BSW biochips were used to directly detect in the fluorescence mode Ang2 at the concentration $10 \mathrm{ng} / \mathrm{ml}$ with an estimated limit of detection of $6.0 \mathrm{ng} / \mathrm{ml}$. Comparison of such a result to our precedent achievements indicates that such a limit of detection of hybrid biochips can be improved by dropping the PtG immobilization step and immobilizing the capture antibodies directly on the ppAA functional layer. This would permit to obtain hybrid biochips with limit of detection below the threshold for Ang2 concentration in healthy patients $(5 \mathrm{ng} / \mathrm{ml})$ and taking advantage of the versatility of the plasma polymerization technique.

\section{Acknowledgements}

This work was funded by the European Commission through the project BILOBA (Grant Agreement 318035). Dr. Paola Rivolo from Politecnico di Torino (Italy) is kindly acknowledged for the deposition of the ppAA layers and for fruitful discussions. Dr. Oleksiy Anopchenko from Baylor University (USA) is acknowledged for the preliminary analysis of the fluorescence data. All partners of the BILOBA project are acknowledged for fruitful discussions.

\section{References}

[1] M. Shinn, W.M. Robertson, Surface plasmon-like sensor based on surface electromagnetic waves in a photonic band-gap material, Sensors Actuators B Chem. 105 (2005) 360-364. doi:10.1016/j.snb.2004.06.024.

[2] P. Yeh, A. Yariv, C.-S. Hong, Electromagnetic propagation in periodic stratified media I General theory*, J. Opt. Soc. Am. 67 (1977) 423. doi:10.1364/JOSA.67.000423.

[3] V.N. Konopsky, E. V Alieva, Photonic Crystal Surface Waves for Optical Biosensors, Anal. Chem. 79 (2007) 4729-4735. doi:10.1021/ac070275y.

[4] M. Liscidini, J.E. Sipe, Analysis of Bloch-surface-wave assisted diffraction-based biosensors, J. Opt. Soc. Am. B. 26 (2009) 279. doi:10.1364/JOSAB.26.000279.

[5] H. Raether, Surface Plasmons, Springer-Verlag, Berlin, 1988.

[6] J. Homola, Surface plasmon resonance sensors for detection of chemical and biological 
species, Chem. Rev. 108 (2008) 462-93. doi:10.1021/cr068107d.

[7] N. Danz, A. Sinibaldi, F. Michelotti, E. Descrovi, P. Munzert, U. Schulz, F. Sonntag, Improving the sensitivity of optical biosensors by means of Bloch surface waves, Biomed. Tech. 57 (2012) 584-587. doi:10.1515/bmt-2012-4246.

[8] R. Rizzo, N. Danz, F. Michelotti, E. Maillart, A. Anopchenko, C. Wächter, Optimization of angularly resolved Bloch surface wave biosensors, Opt. Express. 22 (2014) 23202. doi:10.1364/OE.22.023202.

[9] J.Y. Ye, M. Ishikawa, Enhancing fluorescence detection with a photonic crystal structure in a total-internal-reflection configuration, Opt. Lett. 33 (2008) 1729-1731.

[10] P.C. Mathias, N. Ganesh, L.L. Chan, B.T. Cunningham, Combined enhanced fluorescence and label-free biomolecular detection with a photonic crystal surface, Appl. Opt. 46 (2007) 2351. doi:10.1364/AO.46.002351.

[11] A. Sinibaldi, C. Sampaoli, N. Danz, P. Munzert, L. Sibilio, F. Sonntag, A. Occhicone, E. Falvo, E. Tremante, P. Giacomini, F. Michelotti, Detection of soluble ERBB2 in breast cancer cell lysates using a combined label-free/fluorescence platform based on Bloch surface waves, Biosens. Bioelectron. 92 (2017). doi:10.1016/j.bios.2017.02.012.

[12] R. Rizzo, M. Alvaro, N. Danz, L. Napione, E. Descrovi, S. Schmieder, A. Sinibaldi, R. Chandrawati, S. Rana, P. Munzert, T. Schubert, E. Maillart, A. Anopchenko, P. Rivolo, A. Mascioletti, F. Sonntag, M.M. Stevens, F. Bussolino, F. Michelotti, Bloch surface wave label-free and fluorescence platform for the detection of VEGF biomarker in biological matrices, Sensors Actuators B. Chem. (2017). doi:10.1016/j.snb.2017.09.018.

[13] P. Munzert, N. Danz, A. Sinibaldi, F. Michelotti, Multilayer coatings for Bloch surface wave optical biosensors, Surf. Coatings Technol. (2016). doi:10.1016/j.surfcoat.2016.08.029.

[14] P. Rivolo, M. Castellino, F. Frascella, S. Ricciardi, Ultra-Thin Plasma-Polymerized Functional Coatings for Biosensing: Polyacrylic Acid, Polystyrene and Their Co- Polymer, in: P.Mandracci (Ed.), Cryst. Non-Crystalline Solids, Intech, 2016: pp. 81-104. doi:10.5772/62899.

[15] T. Sfez, E. Descrovi, L. Yu, M. Quaglio, L. Dominici, W. Nakagawa, F. Giorgis, H.P. Herzig, T. Sfez, E. Descrovi, L. Yu, M. Quaglio, L. Dominici, W. Nakagawa, F. Michelotti, F. Giorgis, H.P. Herzig, Two-dimensional optics on silicon nitride multilayer : Refraction of Bloch surface waves Two-dimensional optics on silicon nitride multilayer : Refraction of Bloch surface waves, 151101 (2010). doi:10.1063/1.3385729.

[16] B.R. Förch, A.N. Chifen, A. Bousquet, H.L. Khor, M. Jungblut, L. Chu, I. Osey-mensah, E. 
Sinner, W. Knoll, Recent and Expected Roles of Plasma-Polymerized Films for Biomedical Applications, Chem. Vap. Depos. 13 (2007) 280-294. doi:10.1002/cvde.200604035.

[17] F. Michelotti, T. Gabler, H. Hörhold, R. Waldhausl, A. Bräuer, Prism coupling in DMOPPPV optical waveguides, Opt. Commun. 114 (1995). doi:10.1016/0030-4018(94)00610-7.

[18] S.D. Choudhury, R. Badugu, J.R. Lakowicz, Directing Fluorescence with Plasmonic and Photonic Structures, Accounts Chem. Res. 48 (2015) 2171-2180. doi:10.1021/acs.accounts.5b00100.

[19] K.L. Mittal, Silanes and Other Coupling Agents, Volume 5, CRC Press, 2009.

[20] N. Danz, A. Sinibaldi, P. Munzert, A. Anopchenko, E. Förster, S. Schmieder, R. Chandrawati, R. Rizzo, R. Heller, F. Sonntag, A. Mascioletti, S. Rana, T. Schubert, M.M. Stevens, F. Michelotti, Biosensing platform combining label-free and labelled analysis using Bloch surface waves, SPIE Opt. + Optoelectron. 9506 (2015) 95060V. doi:10.1117/12.2178444.

[21] R. Rizzo, M. Alvaro, N. Danz, L. Napione, E. Descrovi, S. Schmieder, A. Sinibaldi, R. Chandrawati, S. Rana, P. Munzert, T. Schubert, E. Maillart, A. Anopchenko, P. Rivolo, A. Mascioletti, F. Sonntag, M.M. Stevens, F. Bussolino, F. Michelotti, Bloch surface wave enhanced biosensor for the direct detection of Angiopoietin-2 tumor biomarker in human plasma, 2017. 